\title{
Inducible Gene Expression Systems in Plants
}

Zhang Zhuang *, Zhang Qianqian *, Zhang Mengmeng, Dong Zijian, Sun Wenwen, Cao Xuesong, Hu Qüan'an

College of Life Sciences, Liaocheng University, Liaocheng, 252000, China

* These authors contributed equally to this work

D Corresponding author email: huquanan@lcu.edu.cn

Plant Gene and Trait, 2020, Vol.11, No.11 doi: $10.5376 /$ pgt.2020.11.0011

Received: 21 Dec., 2020

Accepted: 22 Dec., 2020

Published: 26 Dec., 2020

Copyright $\odot 2020$ Zhang et al., This article was first published in Molecular Plant Breeding in Chinese, and here was authorized to translate and publish the paper in English under the terms of Creative Commons Attribution License, which permits unrestricted use, distribution, and reproduction in any medium, provided the original work is properly cited.

Preferred citation for this article:

Zhang Z., Zhang Q.Q., Zhang M.M., Dong Z.J., Sun W.W., Cao X.S., and Hu Q.A., 2020, Inducible gene expression systems in plants, Plant Gene and Trait, 11(11): 1-14 (doi: 10.5376/pgt.2020.11.0011)

\begin{abstract}
Gene expression is one of the major applications in plant molecular biology, which plays important role in theoretical and agricultural application researches. Due to the weak foundation and lack of tools, most of the early studies used constitutive promoters, such as the most commonly used $35 \mathrm{~S}$ promoter of cauliflower mosaic virus, to overexpress. With the further researches, people have more understanding of the molecular biology of gene expression, and then derived better and more tools. In order to have spatiotemporal and quantitative fine-tune expression, several inducible gene expression systems have been developed. Classified according to different inducers, the commonly used expression systems are as such, the tetracycline induced, the steroid induced, the ethanol induced, the insecticide induced, and the copper induced. These systems have different working principles because of the different factors and components used; at the same time, there are also commonalities in each of them, they are usually composed of two elements, the activation element and the response element. In this paper, several plant inducible expression systems are reviewed, and their characteristics are analyzed, reviewed and prospected.
\end{abstract}

Keywords Plant gene expression; Inducible expression; Transcription factor; Activator; Responder

Early in the 1980s, constitutive promoters were served to study plant gene expression. Among them, the $35 \mathrm{~S}$ promoter (Odell et al., 1985) from cauliflower mosaic virus (CaMV) and the Nos promoter (Marton et al., 1979) of carmine synthase gene from Agrobacterium tumefaciens Ti plasmid are the most widely used. Its disadvantages are obvious, such as the continuous expression of foreign genes, sometimes have harmful effects on the normal growth of plants. The application of tissue-specific promoter overcomes this disadvantage to some extent, but it is still difficult to accurately control gene expression. With the development of genetic engineering technology, the use of inducible promoters to control target genes transcription has become a new choice. Compared with constitutive and tissue-specific promoters, inducible promoters have the following advantages: 1. without inducer, the system cannot start transcription, thus do not express the target gene; 2 . with inducers, promoters can be activated, transcribed and expressed in specific plant growth periods, specific tissue cells or special environmental conditions; 3 . It is also possible to remove the inducer, to stop the transcription of the target gene(Gatz and Lenk, 1998).

In recent years, there are many researches and discussions on inducible expression systems, among which the chemical induction system, through which precise regulation can be achieved, is most widely used (Zuo and Chua, 2000).

There are three mechanisms by which plant genes can be induced (Hu, 2003), namely, de-inhibition, inactivation, and activation. A variety of induced expression systems have been constructed by using these three mechanisms. The idea of the successfully constructed inducible expression system is basically the same, that is, expression of two separate genes are controlled by suitable respective promoters, one gene encodes the activation protein (transcriptional inhibitor or activator), and the other gene expresses the foreign target gene. Therefore, the induced expression system generally consists of two necessary elements. the first element is used 
to express an artificially constructed chimeric transcription factor, which can specifically bind to a strictly controlled promoter. and the promoter can be activated only after induction, which is generally referred to as a driving element or an activating element. The second element contains the binding site of the transcription factor expressed by the activating element, which is activated to regulate the expression of the target gene, which is generally referred to as a reporter element or a response element. Activating elements can work under the control of the CaMV35S promoter to obtain strong and universal expression, and can also work under the control of developmental stage specific or tissue-specific promoters for local expression. The response element usually contains a smallest CaMV35S promoter, mini35S, so that it can bind to endogenous transcriptional mechanism. The activating element and response element can be different parts of the same DNA sequence to form a unitary induced expression system, or they can be located in different expression cassettes of two different vectors, to form a binary induced expression system after co-transformation of plants. In the early researches, the unitary induced expression system is widely used. With the development of technology, the binary induced expression system is more widely used.

\section{Tetracycline (Tc) Induced Expression System}

\subsection{Tc-on system}

E. coli transposon Tn10 encodes a tetracycline inhibitory protein TetR (Tet Repressor). Tetracycline operon contains a manipulative sequence in the promoter region (Operator; tetO). In the absence of tetracycline, TetR protein can bind to tetO, so that the promoter cannot initiate the transcription of tetracycline resistance gene (Chopra and Roberts, 2001). When tetracycline is added, the TetR protein specifically binds to the tetracycline molecule and changes its structure, resulting in its separation from tetO, thus releases the expression the tetracycline resistance gene. Using this principle, the researchers constructed Tc-on system and Tc-off system, respectively.

Gatz and others established a tetracycline induced expression system and successfully transferred it into plants. In the activation element of the system, they used the CaMV35S promoter to control the expression of TetR protein; In the response element, they constructed a chimeric tetO-35s promoter, and used the promoter to control the expression of $\beta$-glucuronidase (GUS) gene (Figure 1).

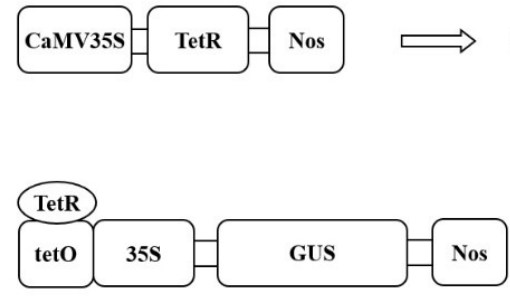

No expression
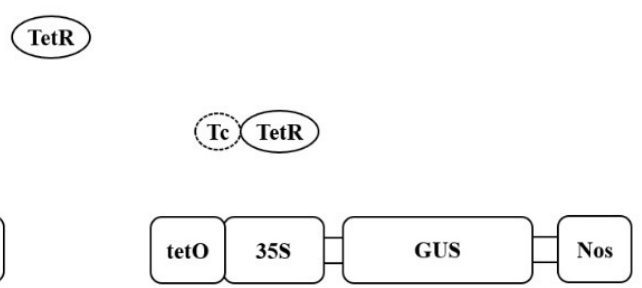

Response element expresses GUS

Expression

Figure 1 Tc-on system

Note: Activation protein TetR is expressed by the constitutive promoter CaMV35S; Nos: carmine synthase Terminator; In the response element, three tetracycline operon sequences tetO were inserted on both sides of $35 \mathrm{~s}$ promoter TATA-box to form a chimeric promoter. Without tetracycline Tc, TetR binding tetO, $\beta$-glucuronidase (GUS) does not express; with Tc, TetR binding $\mathrm{Tc}$, and separates from tetO, releases GUS expression

Using this system, the plants with high expression of TetR protein were successfully obtained. When there is lack of tetracycline in the plant growth environment, the over-expressed TetR combines with tetO, to prevent GUS gene expression, and thus GUS activity could not be detected. When the plants are treated with $0.1 \mathrm{mg} / \mathrm{L}$ tetracycline, TetR binds tetracycline, releases tetO, thus leads to GUS expression and gives detectable GUS activity (Gatz et al., 1992; Gatz and Quail, 1988). 
Tc-on system has been successfully used in tobacco, tomato and potato (David and Perrotrechenmann, 2001; De Veylder et al., 2000). The advantages of the system are as follows: 1. Low background activity and high inductive activity; 2. The amount of inducer is small and easy to be absorbed; 3. It can be used for the study of local and instantaneous expressions, respectively. The disadvantages of the system are as follows: 1 . It cannot be used in the plant model organism arabidopsis thaliana, which may be because high level of TetR has serious effects on the root development of the plant. 2. Tetracycline has a short half-life and requires frequent supplementation of fresh tetracycline (Zuo and Chua, 2000); 3. Tetracycline is an antibiotic and cannot be used in field experiments.

\subsection{Tc-off system}

Similar to the Tc-on system, this system also takes advantage of the characteristics between TetR and its operon sequences. In the Tc-off system, the activation element expresses a tetracycline trans-activating protein (tTA), which has both the DNA-binding activity of TetR protein and the transcriptional activation activity of herpes virus protein (VP16) (De Veylder et al., 2000). In the response element, seven operon sequences are combined with mini35S to form a chimeric promoter TOP10, that is placed upstream of the green fluorescent protein (GFP) gene to regulate gene expression (Figure 2).

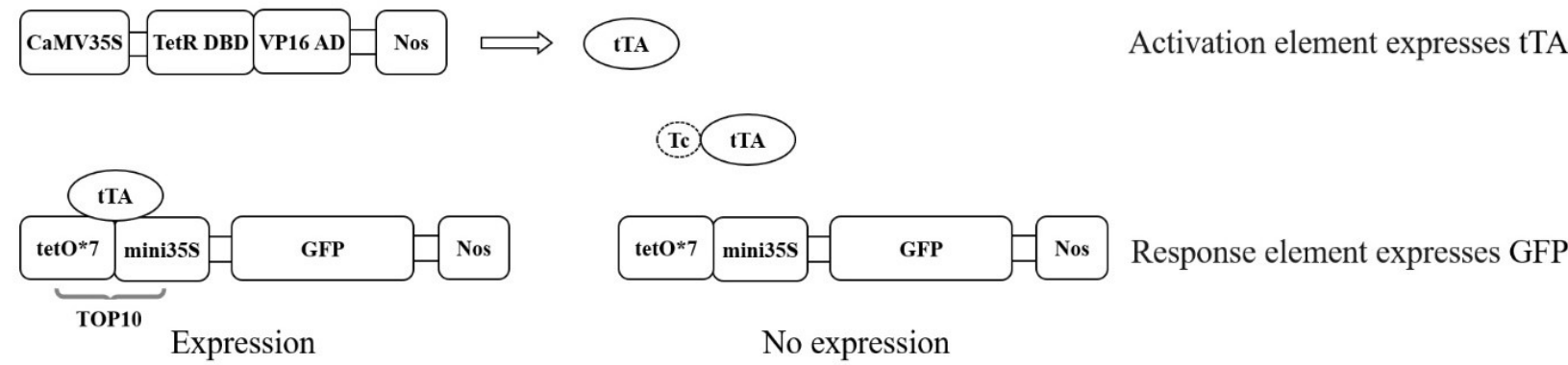

Figure 2 Tc-off system

Note: The activating protein tTA is expressed by the fusion expression of DNA binding domain (DBD) of TetR repressor and the activation domain (VP16 AD) from herpes simplex virus protein 16, and is expressed by CaMV35S promoters; Nos: carmine synthase Terminator; seven tetracycline operon and mini35S fusion TOP10 promoters regulate downstream GFP gene expression; in the absence of tetracycline Tc, tTA binds tetO, very close to TOP10 TATA-box, activated GFP expression. With existence of $\mathrm{Tc}$, and tTA is separated from tetO, expression of GFP is turned off

When there is no tetracycline in the environment, tTA protein can bind to the operon. Using VP16 AD to activate TOP10 promoter and transcribing downstream green fluorescent protein gene (GFP), fluorescence activity thus can be detected. With the addition of tetracycline, tetracycline molecules specifically bind to tTA protein, resulting in dissociation of tTA protein from operon, termination of transcription, and cessation of downstream target gene expression (Weinmann et al., 1994). Studies have shown that tetracycline in $100 \mathrm{ng} / \mathrm{mL}$ is sufficient to inhibit this system.

This system has been successfully used in Tobacco, Arabidopsis (Love et al., 2000) and Mosses (Zeidler et al., 1996). The characteristic of this system is that it can accurately regulate the transcription of genes and thus compare the effects of different transcriptional levels of foreign genes on phenotypes (Gil and Green, 1996). The advantages of the system are as follows: 1. Low background expression; 2. Can accurately control the opening and closing of the target promoter, can carry out quantitative analysis; 3 . Compared with Tc-on system, there is no need to express too many regulatory proteins, which avoids the adverse effects of TetR protein on host plants, and thus can be used in arabidopsis thaliana. The disadvantage of this system is that the system is a negative regulation system, which needs to maintain a certain level of tetracycline concentration in order to keep the target promoter closed, which reduces the practicability of the system. 


\section{Steroid Induced Expression System}

\subsection{Dexamethasone induced GR fusion system}

In mammals, the molecular mechanism of steroid nuclear receptor regulation is highly conservative. For example, the glucocorticoid receptor (GR) interacts with heat shock protein (HSP90) to form an inactive complex in the absence of steroid ligands. This complex is thought to interfere with the interaction of transcription factors with other proteins or DNA targets through steric hindrance. In the presence of steroids, the binding of steroids to the GR ligand binding domain (LBD) leads to the dissociation of the latter from the HSP90 complex, and transcription factors can activate transcription normally (Picard, 1993). There is no similar hormone system in plants, so in many studies, steroid transactivation systems are used to regulate gene expression, such as dexamethasone induction system based on glucocorticoid receptor, such as GVG/UAS system and pOp6/LhGR system, and $\beta$-estradiol induction system based on estrogen receptor (ER).

GR fusion system is the simplest dexamethasone induction system. The strategy of this system is to directly fuse GR and transcription factors to obtain steroid-induced chimeric transcription factors (Schena et al., 1991), which can be directly used to study transcription factors regulating leaf morphogenesis, floral organ determination and lateral organ development in plants (Kirch et al., 2003). The advantage of this system is that the strategy is simple and easy to use and directly express chimeric transcription factors.

\subsection{Dexamethasone induced GVG/UAS system}

Aoyama and Chua constructed the GVG/UAS system based on the principle of glucocorticoid receptor. The activating element constructed by this system expresses an acting protein GVG protein. GVG contains yeast transcription factor GAL4 DBD, VP16 AD and GR LBD (Aoyama and Chua, 1997). The target promoter of the response element consists of a UAS activation region composed of six GAL4 binding sequences and mini35S fusion, which regulates the expression of the target gene (Figure 3).

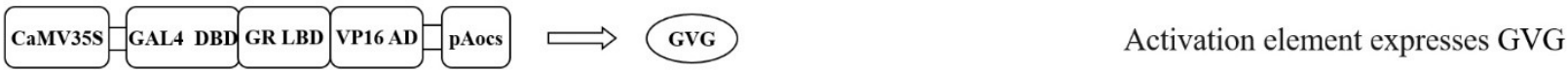

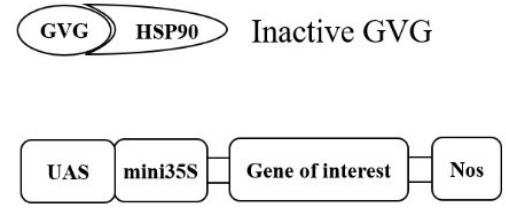

No expression

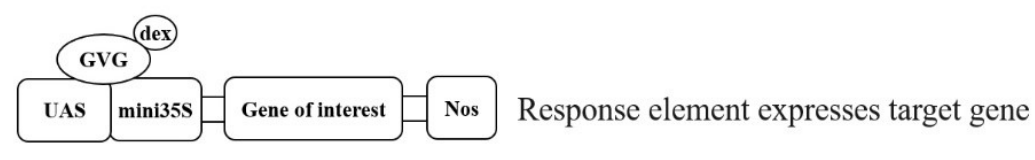

Expression

Figure 3 Dexamethasone induced GVG/UAS system

Note: The acting protein GVG is expressed by the fusion expression of yeast GAL4 transcription factor DNA binding domain (GAL4 DBD) with mouse glucocorticoid receptor ligand binding domain (GR LBD) and herpes simplex virus protein 16 activation domain (VP16 AD). The CaMV35S promoter controls the constitutive expression of GVG; the pAocs: Terminator; the target promoter is composed of UAS activation region and mini35S fusion, the earlier is composed of six GAL4 binding sequences, mini35S to regulate downstream target gene expression. In the absence of dexamethasone dex, GVG binds to the regulatory protein HSP90 in an inactive state; adding dex, GVG binds dex and thus changes the conformation and removes the HSP90, GVG binding UAS sequence to activate the expression of the target gene

Aoyama and Chua, and others showed that GVG protein was constitutively expressed. In the absence of dexamethasone (dex), GVG protein is inactive and foreign genes are not expressed; in the presence of dexamethasone, GVG protein binds to dexamethasone, GVG protein dissociates to active state, binds to UAS activation region, and foreign genes are expressed. Masashi and others (Mori et al., 2001) built an efficient expression system of foreign gene mRNA in transgenic plants using GVG system. The system has been successfully applied in tobacco and Arabidopsis thaliana, but the system itself has some defects. When 
Arabidopsis thaliana and other plants accumulate too much GVG proteins, normal plants growth is affected, seriously and even lethal (Kang et al., 1999). Other studies have shown that the system will affect the expression of some defense-related genes in host plants.

\subsection{Dexamethasone induced pOp6/LhGR system}

SamalovaM and BrzobohatyB established pOp6/LhGR system based on glucocorticoid receptor (Samalova et al., 2005). The activating element of this system expresses LhGR protein, which is the fusion product of the high affinity DNA binding mutant lacI His17 DBD, transcriptional activation domainII of GAL4 and GR LBD. The pOp6 chimeric promoter contains six lac operons upstream of the mini35S promoter to regulate the expression of downstream target genes (Figure 4).

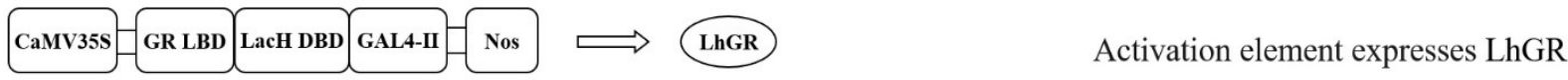

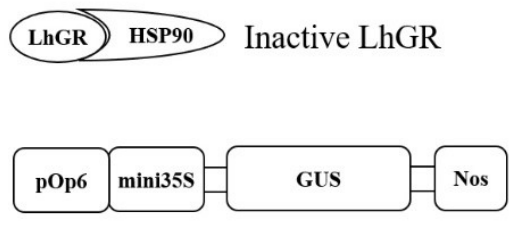

No expression

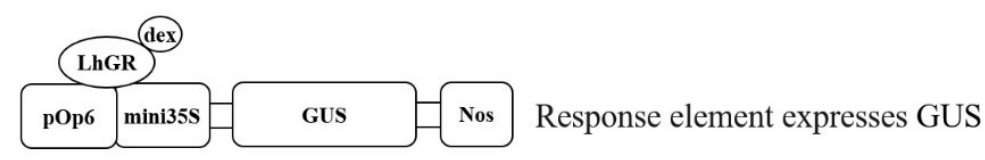

Expression

Figure 4 Dexamethasone induced pOp6/LhGR system

Note: The acting protein LhGR is expressed by the fusion expression of DNA binding domain (LacH DBD) of Saccharomyces cerevisiae lac inhibitor mutant with mouse glucocorticoid receptor ligand binding domain (GR LBD) and yeast transcription factor GAL4 transcriptional activation domain (GAL-II). CaMV35S promoter controls the constitutive expression of LhGR; Nos: carmine synthase Terminator; the target promoter is the fusion expression of six lac operon and mini35S to regulate downstream GUS gene expression. In the absence of dexamethasone dex, LhGR binds to the regulatory protein HSP90 in an inactive state, adding dex, LhGR binds to dex to change the conformation, breaking away from the HSP90, LhGR binds to pOp6 sequence to activate GUS expression

POp6/LhGR system provides a highly sensitive chemically induced transgenic expression system for tobacco, and this system may be suitable for many other plants (Moore et al., 2006). In addition, the system is compatible with previously reported LhG4 systems (Moore et al., 1998); for LhG4 systems, there are a large number of tissue-specific activation mutants available. Therefore, by crossing with appropriate LhGR or LhG4 activation lines, the gene expression can be accurately regulated in time or space by introducing the target gene into tobacco under the control of pOp6 promoter.

\section{4 $\beta$-estradiol induced ER-C1 system}

Bruce and others established a $\beta$-estradiol induction system. The construction strategy of the system is to express an acting factor ER-C1 protein through the activation element, which is expressed by the fusion of the trans-activation domain (AD) of corn activator $\mathrm{C} 1$ and the human ER activation domain (LBD). The target promoter in the response element is comprised of 4 copies of ER element (ERE) and mini35S promoter, which regulates the expression of downstream luciferase (LUC) gene (Figure 5).

Bruce and others verified that the system can be applied to maize BMS (Black Mexican Sweet) cell line (Bruce et al., 2000). Before induction, ER-C1 protein was inactive and could not bind to ERE activation and transcription of LUC gene. After induction with estradiol, ER-C1 protein combine with ERE to activate transcription of downstream luciferase gene. Two days after induction, 14000 relative light unit luciferase activity was detected. The advantage of this induction system is that the amount of inducer is low and there is no obvious toxic effect on plants, but there is no report on the application of this system to transgenic plants. 


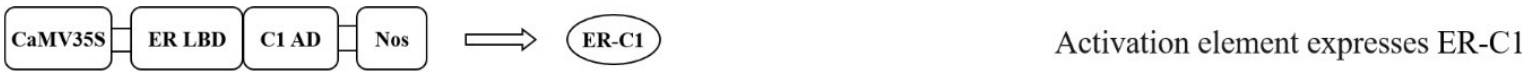

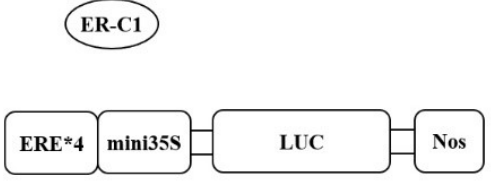

No expression

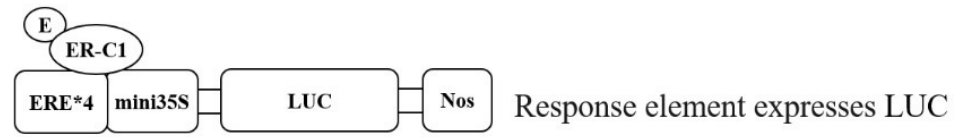

Expression

Figure $5 \beta$-estradiol induced ER-C1 system

Note: The acting protein ER-C1 is expressed by the fusion of human estrogen receptor activation domain (ER LBD) and maize activator $\mathrm{C} 1$ transactivation domain $(\mathrm{C} 1 \mathrm{AD})$. The constitutive expression of ER-C1 is controlled by CaMV35S promoter...Nos: carmine synthase Terminator, and the target promoter is the fusion expression of four ERE elements and mini35S, which regulates the expression of downstream LUC gene. In the absence of $\beta$-estradiol (E), ER-C1 could not bind to ERE elements and luciferase (LUC) could not be expressed, while the conformational change of E-binding to ERE elements could activate the expression of LUC with the addition of Erector

\section{$2.5 \beta$-estradiol induced XVE/OlexA system}

Zuo and others established the XVE/OlexA induction system (Zuo et al., 2000a). The activating element of the system expresses the acting protein XVE, which consists of DBD from the LexA protein of E.Coli, VP16 AD and ER LBD. The target promoter in the response element consists of an 8-copy LexA binding site (OlexA) and mini35S promoter, which regulates the expression of downstream green fluorescent protein gene (Figure 6).

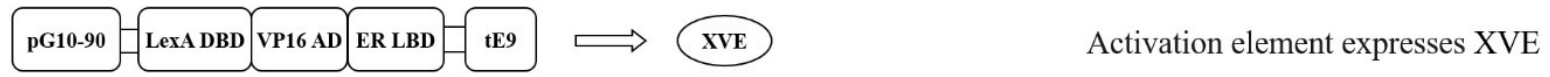

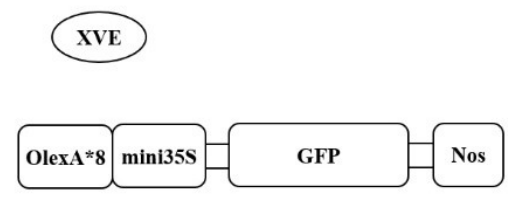

No expression

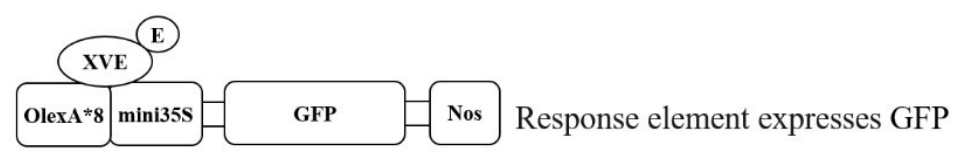

Expression

Figure $6 \beta$-estradiol induced XVE/OlexA system

Note: The acting protein XVE consists of three fusion parts, namely, the DNA binding region (LexA DBD) of E. coli LexA protein, the transcriptional activation region (VP16 AD) of herpes simplex virus protein 16 and the constitutive expression of XVE controlled by the human estrogen receptor regulatory region (ER LBD), pG10-90 promoter; the Nos: carmine synthase Terminator; the target promoter is the fusion expression of eight LexA binding sites (OlexA) and mini35S to regulate the downstream GFP gene expression. In the absence of $\beta$-estradiol (E), XVE could not bind to OlexA elements and GFP could not express; while the addition of $\mathrm{E}, \mathrm{XVE}$ combined with $\mathrm{E}$ to change the conformation could activate GFP expression by binding to OlexA elements

In transgenic Arabidopsis thaliana (Zuo et al., 2002) and tobacco, there was no expression of green fluorescent protein without induction. After induction with estradiol, the expression of green fluorescent protein was activated, and the expression level of GFP was 3-5 times than that of CaMV35 promoter. The advantage of this system is that compared with GVG/UAS system, $\beta$-estradiol inducer has no obvious toxic effect on plants and does not affect the expression of endogenous defense-related genes in host plants (Zuo et al., 2000b). The disadvantage is that the system cannot be used in plants containing plant steroids such as soybeans. 


\section{Binary Control TGV System}

Bohner and others established a binary TGV regulation system (Bohner et al., 1999), which overcomes the limitations of Tc-on and Tc-off systems, but combines the advantages of both of them. The activation element of the system expresses a chimeric transcriptional activator TGV protein, which is composed by three components, namely, TetR DBD, GR LBD and VP16 AD; in the response element, the target gene expression is controlled by the TOP10 promoter (Figure 7).

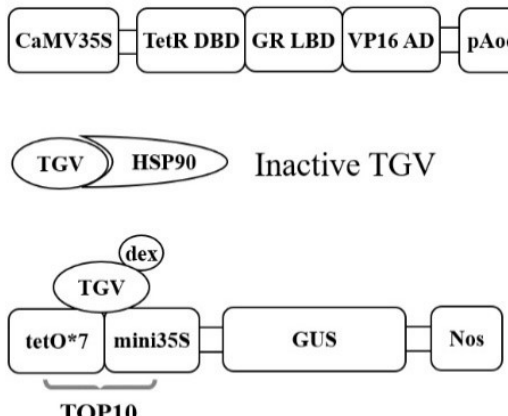

Expression

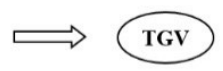

Activation element expresses TGV

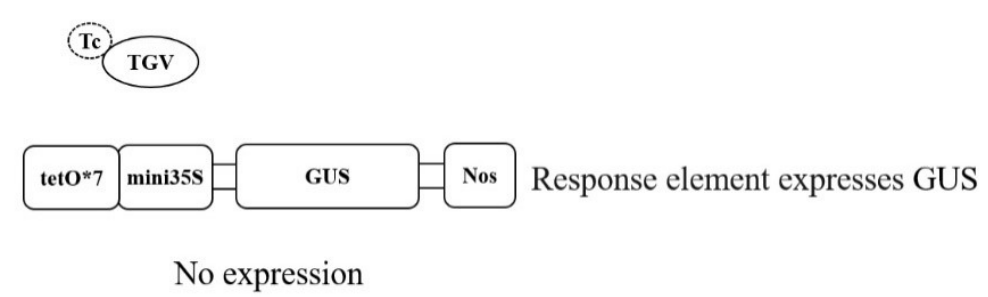

Figure 7 Dexamethasone-tetracycline binary induced system

Note: The acting protein TGV is expressed by the fusion expression of TetR repressor DNA binding domain (TetR DBD) with mouse glucocorticoid receptor ligand binding domain (GR LBD) and herpes simplex virus protein 16 activation domain (VP16 AD). The CaMV35S promoter controls the constitutive expression of TGV; the Nos: carmine synthase Terminator; the target promoter is a TOP10 promoter fused with seven tetracycline operators and mini35S to regulate the expression of downstream GFP gene. In the absence of dexamethasone dex, TGV binds to the regulatory protein HSP90, and thus was inactive. With dex, TGV binds dex and was removed from the HSP90, TGV binds to promoter TOP10 to activate reporter gene expression. While removing Dex plus adding tetracycline Tc, TGV protein was dissociated from TOP10 and transcribed back to uninduced level

The system can be regulated by both tetracycline and dexamethasone. In the absence of dexamethasone, TGV binds to regulatory protein HSP90, and thus is inactive; In the existence of dexamethasone, TGV binds to dexamethasone and thus separated from HSP90, TGV binds to TOP10 promoter and thus activate the expression of $\beta$-glucuronidase gene. After removing dexamethasone and adding tetracycline, TGV protein was dissociated from TOP10, transcription back to uninduced level. The advantages of the system are as follows: 1 . The transcription and termination of foreign genes are regulated by two inducers; 2 . The concentration of inducer required is low; 3 . The newly constructed target promoter does not have the problem caused by tetO methylation. The deficiency of this system is that there is a low level of background expression in BY2 cell line.

\section{Insecticide Induced Expression System}

\subsection{Tebufenozide induced GVHvEcR system}

In practical application, tetracycline and sterol inducers are not suitable for large-scale field applications. Researchers have developed several pesticide induction systems based on insect ecdysone receptor (EcR), which are suitable for field trials.

Martinez and others constructed the GVHvEcR system (Martinez et al., 1999), in which the activating element protein GVHvEcR is expressed, which contains the GR DNA transactivation domain (GR Act), GR DBD, VP16 AD and Heliothisvirescens EcR LBD(HvEcR). The target promoter in the response element contains 6 copies of glucocorticoid response element (GRE) and mini35S promoter. The reporter gene is the $\beta$-glucuronidase (UidA) gene (Figure 8). 


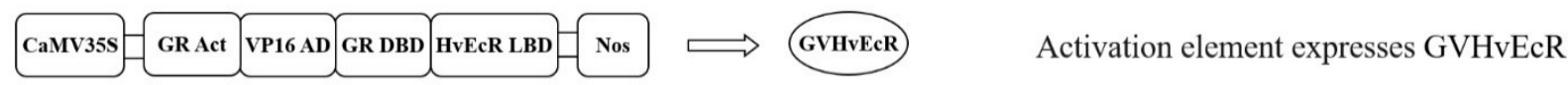

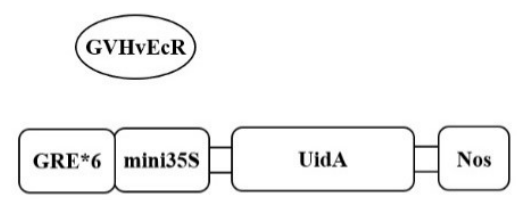

No expression

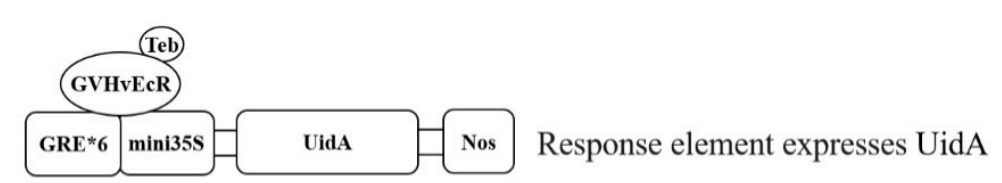

Expression

Figure 8 Tebufenozide induced GVHvEcR system

Note: The acting protein GVHvEcR is fused by the DNA transactivation domain and DNA binding domain (GRAct; GR DBD) of mammalian glucocorticoid receptor, the transcriptional activation domain (VP16 AD) of herpes simplex virus protein 16 and the ligand binding domain (HvEcR LBD) of (Heliothisvirescens) ecdysone receptor in Helicoverpa armigera. CaMV35S promoter controls the constitutive expression of GVHvEcR; Nos: carmine synthase Terminator. The target promoter is the fusion expression of six glucocorticoid response elements (GRE) and mini35S, which regulates the expression of downstream $\beta$-glucuronidase (UidA) gene. In the absence of Tebufenozide (Teb), GVHvEcR could not bind to OlexA element and UidA could not express, and the addition of Teb,GVHvEcR could activate UidA expression by binding to GRE element

Tebufenozide is a non-steroidal ecdysone antagonist, which is often used as a Lepidoptera-specific insecticide in crops. The transcription of the target promoter is induced by the insecticide. In transgenic tobacco, the expression of $\beta$-glucuronidase induced by this insecticide is equivalent to $150 \%$ of the activity of CaMV35S promoter. The advantage of this system is that it is suitable for field use and has no toxicity to plants. The main deficiency of this system is that the foreign gene has a high level of background expression.

\subsection{Methoxyfenozide induced GVEcR system}

Padidam and others developed a system based on spruce EcR (Padidam et al., 2003), The chimeric factors containing EcR LBD, GAL4 or LexA DBD and VP16AD were constructed. In the presence of methoxyfenozide, the activating factor activates the expression of luciferase gene, which is controlled by a chimeric promoter containing a GAL4 or LexA response element and a mini35S promoter (Figure 9).

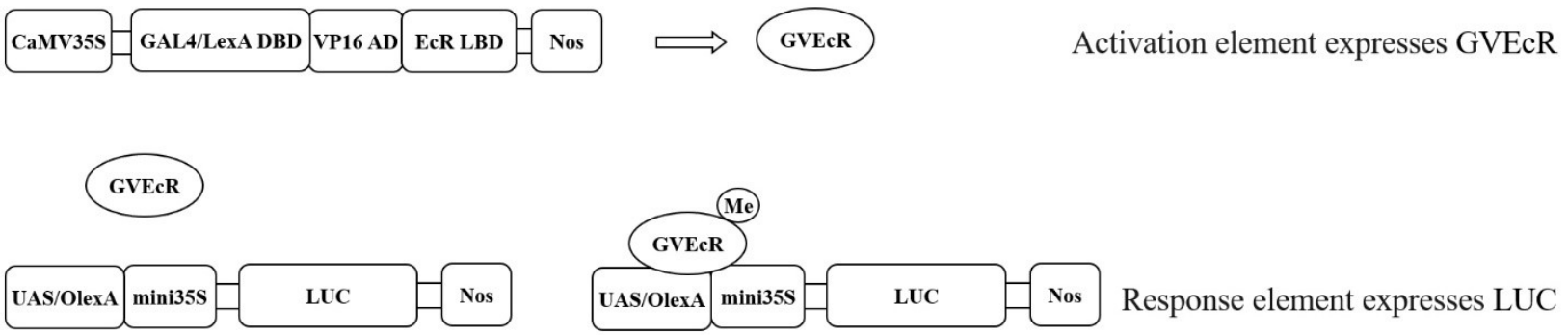

No expression

Expression

Figure 9 methoxyfenozide induced GVEcR system

Note: The acting protein GVEcR is fused by yeast GAL4 transcription factor or Escherichia coli LexA protein DNA binding domain (GAL4/LexA DBD), herpes simplex virus protein 16 transcriptional activation domain (VP16 AD) and spruce ecdysone receptor hormone regulatory domain (EcR LBD). CaMV35S promoter controls the constitutive expression of GVEcR; Nos: carmine synthase Terminator. The target promoter is the fusion expression of GAL response element (UAS) or LexA response element (OlexA) and mini35S, which regulates the expression of downstream luciferase gene (LUC). In the absence of methoxyfenozide (Me), GVEcR could not bind to the target promoter and LUC could not express, and the addition of Me,GVEcR could activate LUC expression by binding to the target promoter 
The expression of low basal and high induced luciferase was optimized by cloning activating elements and reporter genes in different tandem directions. Padidam and others did not observe the abnormal effect of methoxyfenozide on plant growth and development. With the further improvement of methoxyfenozide induction system, EcR from different species of insects can be used to synthesize corresponding inducers, and then multiple induction systems that regulate several genes independently can be developed.

\section{Ethanol Induced AlcR/AlcA System}

In Aspergillus nidulans, transcription factor AlcR controls the expression of AlcA gene, and the expression product of AlcA gene is a kind of ethanol dehydrogenase (Felenbok, 1991). Michael and others constructed the AlcR/AlcA expression system based on this principle (Salter et al., 1998). The activation element of the system is controlled by the CaMV35S promoter, and the target promoter in the response element is the fusion of AlcA and mini35S, which regulates the transcription of chloramphenicol acetyltransferase (CAT) gene (Figure 10).

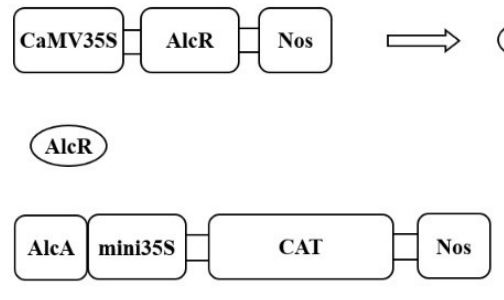

No expression
AlcR Activation element expresses AlcR

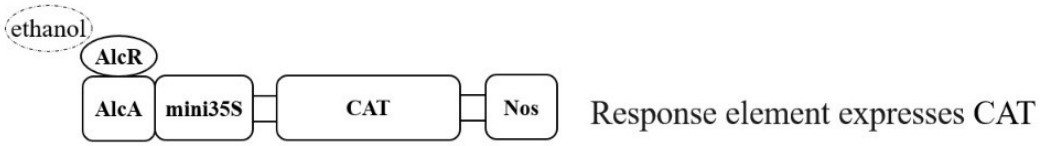

Expression

Figure 10 Ethanol induced AlcR/AlcA expression system

Note: CaMV35S promoter group expressed transcription factor AlcR; AlcA promoter fused with mini35S promoter to form target promoter; Nos: carmine synthase Terminator; without ethanol, transcription factor AlcR could not bind to AlcA promoter and CAT not express; in the presence of ethanol, transcription factor AlcR could bind to AlcA promoter and activate CAT expression

The advantages of the system are as follows: 1 . The inducer is cheap and easy to degrade; 2 . The system construction strategy is very simple (Roslan et al., 2001); 3. The induction response is fast and suitable for field application. The disadvantages of the system are as follows: 1. Ethanol is unstable and volatile; 2. Plants produce ethanol under hypoxia, resulting in foreign gene expression (Deveaux et al., 2003).

The induction system is very suitable for local induction. Tissue-specific promoters can be used to control the expression location of AlcR, and ethanol can be used to quickly induce the expression of foreign genes (Maizel and Weigel, 2004). The system has been successfully applied in plants such as potato, tomato and rape (Sweetman et al., 2002), and has a good application prospect in field experiments.

\section{Copper Induced ACE1 System}

Mett and others (Mett et al., 1996) described a plant gene expression system based on the regulation mechanism of yeast metallothionein (MT) gene (Wright et al., 1988). In this system, CaMV35S controls the expression of ACE1, which encodes a metal-responsive transcription factor. In the presence of copper ions, the ACE1 protein changes its conformation and binds to the metal regulatory element (MRE), and activates the target gene transcription through a chimeric promoter composed of mini35S and six metal regulatory elements (Figure 11).

In order to detect the function of the system in plants, Mett and others prepared a vector containing GUS reporter gene under the control of chimeric promoter, and obtained transgenic tobacco plants. The results showed that whether $50 \mu \mathrm{M} \mathrm{CuSO}_{4}$ was added to the nutrient solution or $0.5 \mu \mathrm{M} \mathrm{CuSO}_{4}$ was sprayed on the leaves, the GUS activity in the leaves of transgenic plants increased by more than 50 times. Another study by Mett and others showed that the copper controllable system constructed with tissue-specific promoters can be used to regulate gene expression in lotus root nodules in a tissue-specific way. 


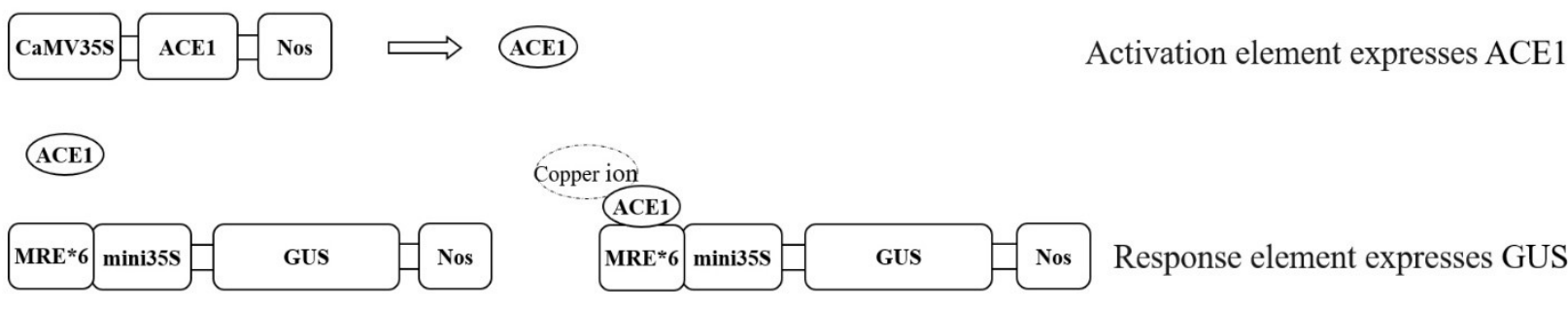

No expression

Expression

Figure 11 Copper induced ACE1 expression system

Note: CaMV35S Promoter Molding expression transcription Factor ACE1; Target Promoter is fused expression of six metal regulatory elements (MRE) and mini35S promoter; Nos: carmine synthase Terminator; In the absence of copper ion, transcription factor ACE1 cannot bind to MRE, GUS is not expressed; in the presence of copper ion, transcription factor ACE1 can combine with MRE, to activate GUS expression

GrangerC and CyrR introduced green fluorescent protein (GFP) gene under the control of copper inducible promoter into Arabidopsis thaliana(Granger and Cyr, 2001), describing the time process of GFP expression up-regulated and down-regulated with copper level, the optimal regulation level of copper, and the tissue-specific types expressed in three transgenic lines. It is suggested that the copper-induced promoter subsystem may be useful in regulating the time and location of gene expression in Arabidopsis thaliana.

\section{Heat Shock Induced System}

The HSP 18.2 gene in Arabidopsis thalian encodes a low molecular weight heat shock protein, its promoter has been successfully used in several plants (Shinmyo et al., 1998), through heat-shock at $37^{\circ} \mathrm{C}$, to induce expression of downstream genes. It is known that the HSP18.2 promoter is suppressed in the absence of heat stress (Matsuhara et al., 2000). Yoshida and others (Yoshida K. et al., 1995) inserted the DNA fragment of $925 \mathrm{bp}$ in the 5 'flanking region of HSP18.2 gene into the upstream of GUS to construct a binary plasmid (Figure 12). The constructed HSP18.2-GUS was introduced into BY-2 cells by electroporation or through Agrobacterium tumefaciens-mediated method to verify that heat shock can induce the expression of HSP18.2 promoter in cultured tobacco cells.

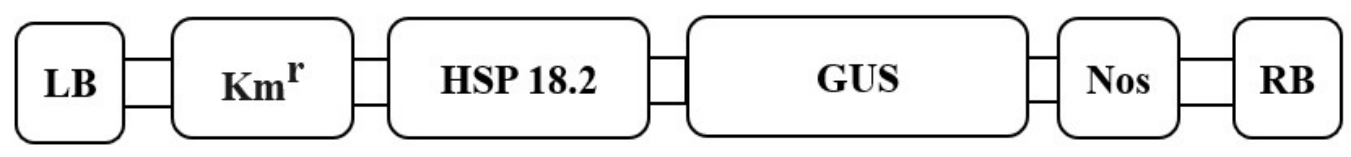

Figure 12 Binary plasmid pGA482-pHSP18.2 T-DNA regional diagram

Note: The heat shock promoter HSP18.2 contains six possible heat shock elements (HSEs); GUS: luciferase gene; Kmr: neomycin phosphotransferase gene; Nos: carmine synthase Terminator; LB/RB: T-DNA left and right boundary sequence

SaidiY and others (Saidi et al., 2005) studied the induction of soybean heat shock Gmhsp17.3B promoter in moss. In stably transformed moss, the expression of Gmhsp $17.3 \mathrm{~B}-$ driven GUS was very low at $25^{\circ} \mathrm{C}$, while non-invasive heat treatment at $38^{\circ} \mathrm{C}$ for a short time could rapidly induce expression of more than 3 orders of magnitude. And the induction level is proportional to the temperature and duration of heat treatment, which proves that the system can fine-tune the expression of protein.

\section{Summary and Prospect}

In the past three decades, the inducible gene expression systems of more than a dozen species of plants have been reported. The practicability of different systems mainly depends on its non-induced expression level and strong induction level. In the ideal system, there is no expression or very low expression in the uninduced state, but in the fully induced state, the expression level should be similar to that of the constitutive strong promoter 
such as CaMV35S. In fact, this requires its regulatory scope to be 1000 times or higher. Equally important is the applicable concentration range of inducers, and the concentration required for complete induction is far less than the concentration that interferes with the normal physiology or development of plants. In addition, artificially constructed chimeric transcription factors should not cause any adverse effects in the induced or non-induced state; the inducible system should also be applicable to a variety of plants, including at least Arabidopsis thaliana and tobacco. So far, the reported systems only partially meet one or more of the above criteria; an ideal system that can accurately induce the expression of target genes in plant organs or tissues and meet all the standards is still the goal of people's expectation and efforts.

At present, worldwide researchers have been committed to the development of new plant inducible expression systems, or to further optimize the existing systems. By using different activation domains and binding domains to construct new chimeric transcription factors, or optimizing the basic promoter and transcription factor binding sequences in chimeric promoters, low background expression or no background expression can be obtained. At the same time, the ideal system should have high level of induced expression. At the same time, with the development of proteomics and systems biology, it is often necessary to study the function of multiple genes at the same time; therefore, a multi-induced expression system which can regulate several genes independently will have a very good application prospect.

\section{Authors' contributions}

$\mathrm{HQ}$ and ZZ designed the article, ZZ and ZQQ collected literature and wrote the primary MS, SWW and ZMM helped ZQQ, DZJ help ZZ. HQ and CXS reviewed and guided the writing and corrections. All authors read and approved the final manuscript.

\section{Acknowledgements}

Thanks to the Natural Science Foundation of Shandong Province (Project No.: ZR2014CM001), the Opening project of the State Key Laboratory of Crop Biology of Shandong Agricultural University (Project No.: 2018KF03) and the Scientific Research start-up Fund of the Ministry of Education of China (2015).

\section{Reference}

Aoyama T., andChua N.,1997, A Glucocorticoid-mediated transcriptional induction system in transgenic plants, Plant Journal, 11(3): 605-612 https://doi.org/10.1046/j.1365-313X.1997.11030605.x PMid:9107046

Bohner S., Lenk I., Rieping M., Herold M., andGatz C., 1999, Transcriptional activator Tgv mediates dexamethasone - inducible and tetracycline inactivatable gene expression, Plant Journal, 19(1): 87-95 https://doi.org/10.1046/j.1365-313X.1999.00503.x PMid: 10417730

Bruce W.B., Folkerts O., Garnaat C.W., Crasta O., Roth B., andBowen B., 2000, Expression profiling of the maize flavonoid pathway genes controlled by estradiol-inducible transcription factors Crc and P., The Plant Cell, 12(1): 65-79

https://doi.org/10.1105/tpc.12.1.65 PMid:10634908 PMCid:PMC140215

Chopra I., andRoberts M.C., 2001, Tetracycline antibiotics: mode of action, applications, molecular biology, and epidemiology of bacterial resistance, Microbiology and Molecular Biology Reviews, 65(2): 232-260

https://doi.org/10.1128/MMBR.65.2.232-260.2001 PMid:11381101 PMCid:PMC99026

David K., and Perrotrechenmann C., 2001, Characterization of a tobacco bright yellow 2 cell line expressing the tetracycline repressor at a high level for strict regulation of transgene expression, Plant Physiology, 125(4): 1548-1553

https://doi.org/10.1104/pp.125.4.1548 PMid:11299335 PMCid:PMC1539379

De Veylder L., Beeckman T., Van Montagu M., AndInze D., 2000, Increased leakiness of the tetracycline - inducible triple - op promoter in dividing cells renders it unsuitable for high inducible levels of a dominant negative cdc2aat gene, Journal of Experimental Botany, 51(351): 1647-1653 https://doi.org/10.1093/jexbot/51.351.1647 PMid:11053453

Deveaux Y., Peaucelle A., Roberts G.R., Coen E., Simon R., Mizukami Y., Traas J., Murray J.A.H., Doonan J.H., and Laufs P., 2003, The ethanol switch: a tool for tissue - specific gene induction during plant development, Plant Journal, 36(6): 918-930 https://doi.org/10.1046/j.1365-313X.2003.01922.x 
Felenbok B., 1991, The ethanol utilization regulon of aspergillus nidulans: the alca-alcr system as a tool for the expression of recombinant proteins, Journal of Biotechnology, 17(1): 11-17 11-17 https://doi.org/10.1016/0168-1656(91)90023-O

Gatz C., Frohberg C., and Wendenburg R., 1992, Stringent repression and homogeneous de - repression by tetracycline of a modified Camv $35 \mathrm{~s}$ promoter in intact transgenic tobacco plants, Plant Journal, 2(3): 397-404 https://doi.org/10.1046/j.1365-313X.1992.t01-37-00999.x PMid: 1303802

Gatz C., and Lenk I., 1998, Promoters That Respond to Chemical Inducers, Trends in Plant Science 3(9): 352-358 https://doi.org/10.1016/S1360-1385(98)01287-4

Gatz C., and Quail P.H., 1988, Tn10-Encoded Tet repressor can regulate an operator-containing plant promoter, Proceedings of the National Academy of Sciences of the United States of America, 85(5): 1394-1397

https://doi.org/10.1073/pnas.85.5.1394 PMid:2830617 PMCid:PMC279777

Gil P., and Green P.J., 1996, Multiple regions of the arabidopsis saur-ac1 gene control transcript abundance: The 3' Untranslated Region Functions as an Mrna Instability Determinant, The EMBO Journal, 15(7): 1678-1686

https://doi.org/10.1002/i.1460-2075.1996.tb00513.x PMid:8612592 PMCid:PMC450079

Granger C.L., and Cyr R.J., 2001, Characterization of the yeast copper-inducible promoter system in Arabidopsis thaliana, Plant Cell Reports, 20(3): $227-234$

https://doi.org/10.1007/s002990000308 PMid:12026935

Hu T.Z., 2003, The Chemical-Inducible Expression System of Plant Genes, Molecular Plant Breeding, 1(5/6): 731-736

Kang H., Fang Y., andSingh K. B.,1999. A glucocorticoid - inducible transcription system causes severe growth defects in arabidopsis and induces defense - related genes, Plant Journal, 20(1): 127-133 https://doi.org/10.1046/j.1365-313X.1999.00575.x PMid:10571872

Kirch T., Simon R., Grünewald M., andWerr W., 2003, The Dornroschen/Enhancer of shoot regeneration1 gene of arabidopsis acts in the control of meristem ccll fate and lateral organ development, Plant Cell, 15(3): 694-705 https://doi.org/10.1105/tpc.009480 PMid:12615942 PMCid:PMC150023

Love J., Scott A.C., and Thompson W.F., 2000, Stringent control of transgene expression in arabidopsis thaliana using the top10 promoter system, The Plant Journal, 21(6): 579-588 579-588 https://doi.org/10.1046/j.1365-313x.2000.00706.x PMid:10758509

Maizel A., and Weigel D., 2004, Temporally and spatially controlled induction of gene expression in Arabidopsis thaliana, Plant Journal, 38(1): 164-171 https://doi.org/10.1111/j.1365-313X.2004.02027.x PMid: 15053769

Martinez A., Sparks C.A., Hart C.A., Thompson J., and Jepson I., 1999, Ecdysone agonist inducible transcription in transgenic tobacco plants, Plant Journal, 19(1): 97-106 97-106 https://doi.org/10.1046/j.1365-313X.1999.00504.x PMid:10417731

Marton L., Wullems G.J., Molendijk L., and Schilperoort R.A., 1979, In vitro transformation of cultured cells from nicotiana tabacum by agrobacterium tumefaciens, Nature, 277(5692): 129-131 https://doi.org/10.1038/277129a0

Matsuhara S., Jingu F., Takahashi T., and Komeda Y., 2000, Heat-shock tagging: a simple method for expression and isolation of plant genome dna flanked by t-dna insertions, Plant Journal, 22(1): 79-86

https://doi.org/10.1046/j.1365-313x.2000.00716.x PMid:10792823

Mett V., Podivinsky E., Tennant A.M., Lochhead L.P., Jones W.T., and Reynolds P.H.S., 1996, A system for tissue-specific copper-controllable gene expression in transgenic plants: Nodule-Specific Antisense of Aspartate Aminotransferase-P2, Transgenic Research, 5(2): 105-113 https://doi.org/10.1007/BF01969428 PMid:8866892

Moore I., Gälweiler L., Grosskopf D., Schell J., and Palme K., 1998, A transcription activation system for regulated gene expression in transgenic plants, Proc Natl Acad Sci USA, 95(1): 376-381 https://doi.org/10.1073/pnas.95.1.376 PMid:9419383 PMCid:PMC18229 
Moore I., Samalova M., and Kurup S., 2006, Transactivated and chemically inducible gene expression in plants, Plant Journal, 45(4): 651-683 https://doi.org/10.1111/j.1365-313X.2006.02660.x PMid:16441354

Mori M., Fujihara N., Mise K., and Furusawa I., 2001, Inducible high - level mrna amplification system by viral replicase in transgenic plants, Plant Journal, 27(1): 79-86 https://doi.org/10.1046/j.1365-313x.2001.01079.x PMid: 11489185

Odell J.T., Nagy F., and Chua N.H., 1985, Identification of DNA sequences required for activity of the cauliflower mosaic virus 35 s Promoter, Nature, 313(6005): 810-812 https://doi.org/10.1038/313810a0 PMid:3974711

Padidam M., Gore M.A., Lu D.L., and Smirnova O., 2003, Chemical-inducible, ecdysone receptor-based gene expression system for plants, Transgenic Research, 12(1): 101-109 https://doi.org/10.1023/A:1022113817892 PMid: 12650528

Picard D., 1993, Steroid-binding domains for regulating the functions of heterologous proteins in cis, Trends in cell biology, 3(8): 278-280 https://doi.org/10.1016/0962-8924(93)90057-8

Roslan H.A., Salter M.G., Wood C.D., White M.R.H., Croft K.P., Robson F., Coupland G., Doonan J.H., Laufs P., and Tomsett A.B., 2001, Characterization of the ethanol-inducible Alc gene-expression system in Arabidopsis thaliana. Plant Journal, 28(2): 225-235 https://doi.org/10.1046/j.1365-313X.2001.01146.x PMid:11722766

Saidi Y., Finka A., Chakhporanian M., Zryd J., Schaefer D.G., and Goloubinoff P., 2005, Controlled expression of recombinant proteins in physcomitrella patens by a conditional heat-shock promoter: A Tool for Plant Research and Biotechnology, Plant Molecular Biology, 59(5): 697-711 https://doi.org/10.1007/s11103-005-0889-z PMid: 16270224

Salter M.G., Paine J.A.M., Riddell K.V., Jepson I., Greenland A.J., Caddick M.X., and Tomsett A.B., 1998, Characterisation of the ethanol-inducible Alc gene expression system for transgenic plants, Plant Journal, 16(1): 127-132 https://doi.org/10.1046/j.1365-313x.1998.00281.x

Samalova M., Brzobohaty B., and Moore I., 2005, Pop6/Lhgr: A stringently regulated and highly responsive dexamethasone - inducible gene expression system for tobacco, Plant Journal, 41(6): 919-935 https://doi.org/10.1111/j.1365-313X.2005.02341.x PMid: 15743454

Schena M., Lloyd A.M., and Davis R.W., 1991, A steroid-inducible gene expression system for plant cells, Proc Natl Acad Sci USA, 88(23): 10421-10425 https://doi.org/10.1073/pnas.88.23.10421 PMid:1961707 PMCid:PMC52940

Shinmyo A., Shoji T., Bando E., Nagaya S., Nakai Y., Kato K., Sekine M., and Yoshida K., 1998, Metabolic engineering of cultured tobacco cells, Biotechnology and Bioengineering, 58(2/3) 329-332 https://doi.org/10.1002/(SICI)1097-0290(19980420)58:2/3<329::AID-BIT34>3.0.CO;2-4

Sweetman J. P., Chu C., Qu N., Greenland A. J., Sonnewald U., and Jepson I., 2002, Ethanol vapor is an efficient inducer of the Alc gene expression system in model and crop plant species, Plant Physiology, 129(3): 943-948

https://doi.org/10.1104/pp.010937 PMid:12114549 PMCid:PMC1540238

Weinmann P., Gossen M., Hillen W., Bujard H., and Gatz C., 1994, A chimeric transactivator allows tetracycline-responsive gene expression in whole plants, Plant Journal, 5(4): 559-569 https://doi.org/10.1046/i.1365-313X.1994.5040559.x PMid: 8012406

Wright C.F., Hamer D.H., and Mckenney K., 1988, Autoregulation of the yeast copper metallothionein gene depends on metal binding, Journal of Biological Chemistry, 263(3): 1570-1574

Yoshida K., Kasai T., Garcia M. R. C., Sawada S., Shoji T., Shimizu S., Yamazaki K., Komeda Y., and Shinmyo A., 1995, Heat-inducible expression system for a foreign gene in cultured tobacco cells using the hsp18.2 promoter of Arabidopsis thaliana, Applied Microbiology and Biotechnology, 44(3): 466-472 https://doi.org/10.1007/s002530050583 PMid: 8597550

Zeidler M., Gatz C., Hartmann E., andHughes J., 1996, Tetracycline-regulated reporter gene expression in the moss physcomitrella patens, Plant Molecular Biology, 30(1): 199-205 https://doi.org/10.1007/BF00017815 
Plant Gene and Trait 2020, Vol.11, No.11, 1-14

http://genbreedpublisher.com/index.php/pgt

Zuo J., and Chua N., 2000, Chemical-inducible systems for regulated expression of plant genes, Current opinion in biotechnology, 11(2): 146-151 https://doi.org/10.1016/S0958-1669(00)00073-2

Zuo J., Niu Q., andChua N., 2000a, An estrogen receptor - based transactivator xve mediates highly inducible gene expression in transgenic plants, Plant Journal, 24(2): 265-273

https://doi.org/10.1046/j.1365-313x.2000.00868.x

PMid:11069700

Zuo J., Niu Q., Frugis G., andChua N., 2002, The wuschel gene promotes vegetative-to-embryonic transition in Arabidopsis, Plant Journal, 30(3): 349-359 https://doi.org/10.1046/j.1365-313X.2002.01289.X

PMid:12000682

Zuo J., Niu Q., Nishizawa N.K., Wu Y., Kost B., and Chua N., 2000b, Korrigan, an Arabidopsis Endo-1,4-B-Glucanase, localizes to the cell plate by polarized targeting and is essential for cytokinesis, The Plant Cell, 12(7): 1137-1152

https://doi.org/10.1105/tpc.12.7.1137

PMid:10899980 PMCid:PMC149055 